\title{
Clustering district heat exchange stations using smart meter consumption data
}

\author{
Tureczek, Alexander Martin; Nielsen, Per Sieverts; Madsen, Henrik; Brun, Adam
}

Published in:

Energy and Buildings

Link to article, DOI:

10.1016/j.enbuild.2018.10.009

Publication date:

2019

Document Version

Peer reviewed version

Link back to DTU Orbit

Citation (APA):

Tureczek, A. M., Nielsen, P. S., Madsen, H., \& Brun, A. (2019). Clustering district heat exchange stations using smart meter consumption data. Energy and Buildings, 182, 144-158.

https://doi.org/10.1016/j.enbuild.2018.10.009

\section{General rights}

Copyright and moral rights for the publications made accessible in the public portal are retained by the authors and/or other copyright owners and it is a condition of accessing publications that users recognise and abide by the legal requirements associated with these rights.

- Users may download and print one copy of any publication from the public portal for the purpose of private study or research.

- You may not further distribute the material or use it for any profit-making activity or commercial gain

- You may freely distribute the URL identifying the publication in the public portal 


\title{
1 Clustering District Heat Exchange Stations Using Smart Meter Consumption Data
}

3

4

5

6

7

8

9 10

Alexander Martin Tureczek ${ }^{a, *}$, Per Sieverts Nielsen ${ }^{a}$, Henrik Madsen ${ }^{b}$, Adam Brun $^{c}$

aSystems Analysis, Management Engineering, Technical University of Denmark, 2800 Kgs. Lyngby, Denmark *atur@dtu.dk, Tel: +45-2346-0989, Produktionstorvet Building 426, room 125.

aSystems Analysis, Management Engineering, Technical University of Denmark, 2800 Kgs. Lyngby, Denmark pernn@dtu.dk

${ }^{b}$ Dynamical Systems, Compute, Technical University of Denmark, 2800 Kgs. Lyngby, Denmark, hmad@dtu.dk

'Business Development, Affald Varme Aarhus, 8210 Aarhus V, Denmark, adbr@aarhus.dk

\begin{abstract}
Contrary to electricity smart meter data analysis, little research regarding district heat smart meter data has been published. Previous papers on smart meter data analytics have not investigated autocorrelation in smart meter data. This paper examines district heat smart meter data from the largest district heat supplier in Denmark and autocorrelation is identified in the data. The K-Means algorithm is not able to take autocorrelation into account when clustering. We propose different data transformation methods to enable KMeans to account for this autocorrelation information in the data by using wavelet transformation and autocorrelation features. Our results show that the K-Means yield acceptable clustering results for district heat data when clustering normalized data, inclusion of autocorrelation improves the clustering. The clusters on normalized data are similar to the wavelet transformed clusters, where the autocorrelation has been accounted for. The clustering achieved with the autocorrelation transformation yields finer clusters through accounting for autocorrelation. We are not able to statistically show a difference between the transformations. All transformations result in shadowing clusters, but the autocorrelation transformation generates fewer shadow clusters and reduce the number of dimensions from 744 to 24 , resulting in a dramatic reduction in $\mathrm{K}$ Means runtime.
\end{abstract}

\section{Highlights}

1. Existence of autocorrelation in the smart meter data is shown.

2. Preprocessing of data enables K-Means to account for autocorrelation.

3. Implementation of cross-validation for unsupervised learning.

4. Autocorrelation feature from input data enable finer clustering from K-Means.

5. Wavelets reduce data with ensuing faster runtime without notable change to clusters.

\section{Keywords}

Clustering; Feature Extraction; Autocorrelation; Wavelet Analysis; Smart Meter Data; Load Pattern; 


\section{Introduction}

In traditional electricity production, fossil fuels and organic waste is burned to generate electricity, the incineration process generates heat as a bi-product. In Denmark, this heat is increasingly utilized as a source for domestic and industrial heating, through district heating systems. Combined heat and power plants (CHP) help cover the demand for electricity and heat of larger populated areas, while smaller heat production plants supply rural districts. Overall District Heating supplies heating and hot water to approximately 64\% [1] of all Danish households.

Since the energy crisis in 1973 district heating has received much attention in Denmark as an efficient and environmentally friendly means to ensure heating. Current research in district heating systems is culminating with 4th generation, where the dominant research focus has been directed towards more efficient technical solutions such as better pipes, flow control, drag minimizing additives and lower temperature in the system. Much less effort has been directed towards the understanding of consumption as part of the system and driver of the demand.

Recent advent of smart meters, for automatic metering of consumption, has enabled consumption recordings at an unprecedented detail, moving from biannual manual readings to automatic hourly readings. Many Danish district heat utilities have embraced this technology and installed smart meters at heat exchange stations, where the transmission and distribution grid transfer energy to individual household areas. Installation of meters at these levels enable the district heat utilities to supervise their system in an intelligent way, but also to increase their knowledge of their consumers for better understanding the demand.

The past decade has witnessed a strong research and technology focus on electricity smart grids where the introduction of smart meters at household level has enabled detailed recording of consumption. Recording of consumption at household level by the minute can help optimize the electricity grid and identify flexibility in the entire system, by way of increased consumer knowledge. Introduction of modern metering equipment for district heating, will in theory, make it possible to do the same consumption pattern analysis, which successfully has been applied to electricity smart meter data in the last decade. This paper will investigate the feasibility of the learnings and methods from smart meter electricity consumption clustering in a district heat setting and assess if these learnings are readily applicable to district heating consumption data.

Heat smart meter data analysis is not only relevant for understanding heat consumption patterns in district heating networks from an academic point of view, but also allow the district heating companies to understand their consumer better and optimize their heat production. We suggest aggregated smart meter district heat consumption data can be used for better evaluation of the demand for heating in an area.

The clustering of consumption at aggregated level enable improvements in production efficiency in the current grid, but the knowledge of consumption clusters also can be used for dimensioning the heat pipes when 
expanding the district heating grid. Both the immediate improvements to production efficiency and savings in capital expenditure through correct dimensioning have the potential to reduce utility costs and thus in turn reduce consumer cost. Furthermore the smart meter district heating consumption data allow for clustering of consumption into more homogeneous sub-clusters, making it feasible for utilities to identify patterns of similar consumption. These clusters are more manageable than individual consumers regarding production efficiency, when expanding the grid and for directed campaigns related to consumption awareness.

This paper makes the following contributions: First, this paper presents analysis of smart meter consumption data at heat exchange station level, including imputation of outliers. Second, the paper confirms the existence of autocorrelation in smart meter data, information which K-Means ignores in the clustering. Third, the paper introduces methods to enable K-Means to account for autocorrelation information in data, by careful transformation of K-Means input data. This can easily be extended to account for other intrinsic data structures. Fourth, the paper extends the notion of cross-validation into unsupervised learning by creating pseudo response variables from cluster validation indices.

The rest of this paper is divided into 5 sections; current relevant literature is listed in section 2, while section 3 describes the data and the preprocessing performed, section 4 discusses the methods applied for clustering. In section 5 the results from clustering using K-means, with and without transformed data are shown. Section 6 discusses the results and their implications while section 7 concludes upon the findings. Finally section 8 includes an executive summary.

\section{Literature Review}

A literature study [2] on smart meter data clustering, which evaluated more than 2000 papers concerning application of energy smart meter data for consumption clustering, identified no papers applying district heating smart meter data for household consumption clustering. Electricity consumption smart metering data have demonstrated significant differences in consumption patterns [3]. This discrepancy can be attributed to differences in lifestyles illustrating the need for better understanding of consumer behavior and consumption patterns, to facilitate more efficient use of resources.

The review [2] also supplies a list of prevalent methods for consumer clustering applying smart meter data. Kmeans is frequently, and thoroughly studied [3-5], and repeatedly compared to more advanced methods such as follow-the-leader [6,7] and hierarchical clustering [8-10]. Experiments with data transformation for preprocessing input data prior to K-Means clustering is conducted in [11]. Evaluation of the resulting clusters is estimated by applying various validation indices [12-14].

There was no clear indication of time interval to evaluate ranging from days [15] to a year [16], and few papers amended external data such as weather data [8], survey information $[17,18]$ and occasionally with energy audits [19] to evaluate consumers and behavior [20]. Only one recent paper which applied smart meter data on 
district heating was identified; [21] applies hourly meter data from district heat substations to evaluate heat load patterns on predefined customer classes.

105

With only few identified studies relating to district heating smart meter data, we will draw much inspiration from research done in electricity smart meter analysis, where K-Means is extensively applied for clustering. The simplicity of the K-Means algorithm has some caveat to which we will discuss solutions. We hypothesize the existence of autocorrelation in the smart meter data. Existence of autocorrelation has consequences for KMeans ability to cluster. Confirmation of the existence of autocorrelation will require circumvention by carefully preprocessing the input data to the K-Means algorithm leaving the K-Means algorithm unaltered.

\section{Data Summary and Preprocessing}

This section describes the data analyzed in this paper, we do so by applying a data description table proposed by [2], presenting important data information in a table view. Furthermore this section will describe the data preprocessing completed while preparing the data for analytics.

The data applied in this study is kindly supplied by AffaldVarme Aarhus (AVA), the largest district heat supplier in Denmark, covering the municipality of Aarhus including suburban and rural areas. Additionally, AVA also supplies a handful of smaller municipalities such as Skanderborg, Odder, Hørning and Hornslet through their transmission grid. The raw data initially comprises hourly readings from the 53 heat exchange stations, which we hereafter refer to as "HX stations". HX stations link the pressurized high temperature water in the transmission grid to the lower temperature distribution grid. The data recordings encompass the time period January $1^{\text {st }} 2017$ till January $31^{\text {st }} 2017$. Three HX stations have been removed from the data set because of incomplete readings. The data description in Table 1 gives an overview of the data set, with the initial and final sample sizes applied in this paper. The structure was proposed in [2] as a standardized means to report minimum data set information to the reader.

\begin{tabular}{|l|l|}
\hline Data Description & Value \\
\hline Type & $\begin{array}{l}\text { Smart meter readings from district heat exchange stations, } \\
\text { exchanging heat from transmission to distribution grid. } \\
\text { Supplying smaller geographical areas of residential and } \\
\text { industrial consumers with heat. }\end{array}$ \\
\hline Country & Denmark \\
\hline Region & Municipality of Aarhus \\
\hline Supplier & AffaldVarme Aarhus (AVA) \\
\hline Initial Data Size & 53 district heat smart meters with 744 observations each. \\
\hline Exclusion of Data & $\begin{array}{l}\text { Meter \#130 was removed as it is a large company Heat } \\
\text { exchange station servicing only 1 customer. }\end{array}$ \\
\hline Missing Values & $\begin{array}{l}\text { Meters: \#118A, \#136J, \#147 discarded du to missing data for } \\
\text { entire January. }\end{array}$ \\
\hline
\end{tabular}




\begin{tabular}{|l|l|}
\hline & $\begin{array}{l}\text { Meters: \#111C, \#119, \#133, \#134, \#135, \#136, \#148 and } \\
\# \mathbf{1 5 1} \text { erroneous readings. These errors were imputed as } \\
\text { described in section 3, and the meters kept in the data set. }\end{array}$ \\
\hline Final Data Size & 49 district heat smart meters with complete data \\
\hline Recording Frequency & Hourly \\
\hline Start & $01 / 01 / 2017$ \\
\hline End & $31 / 01 / 2017$ \\
\hline Length & $\begin{array}{l}\text { 744 recordings per meter. Hourly recording for entire } \\
\text { January. }\end{array}$ \\
\hline Referral & Data never before referenced. \\
\hline
\end{tabular}

Table 1 - Data description as proposed in [2]. The table produces an overview of the data utilized in this study; how many smart meter readings are included in the final data set and which smart meters were disregarded for what reason.

131

132

133

134

135

136 
137 The data set from AVA contains, as shown in Table 1, meter readings from January $1^{\text {st }}, 2017$ until January $31^{\text {st }}$, 138 2017, yielding 744 recordings per HX station. January is one of the coldest months in Denmark and thus a 139 period where the HX stations are very active the entire day and month as most customers demand heat.

140 The HX meter readings utilized in this study are complete for most readings. Three HX stations (118A, 136J, 141 147) have missing values for the entire January, and are removed from the dataset; the three $H X$ stations are 142 one mobile HX station, and $2 \mathrm{HX}$ stations supplying an external utility company. Finally, HX station 130, was removed as it is a station supplying only one company and hence not representative as an HX station.

Meters 111C, 119, 133, 134, 135, 136, 148 and 151 all experienced faulty readings, seen as spikes or outtakes in Figure 1 (left). The erroneous readings are easily identified visually, but also via data, as they all have zeroconsumption readings. Furthermore a few of the meters exhibit sudden very high consumption readings. There are several scenarios which can explain the erratic readings; service stops, cold water pockets, or pipe failure. AVA registered none of those in the given period hence we treat the outliers as meter misreading. Rectification of the outliers is done by imputing the series mean value onto each outlier. This is a simple technique and proved very successful with the current data as can be visually inspected in Figure 1 (right) where the error mean corrected series are shown. All spikes and breaks in the meter readings have been evened out without inducing noticeable artifacts.
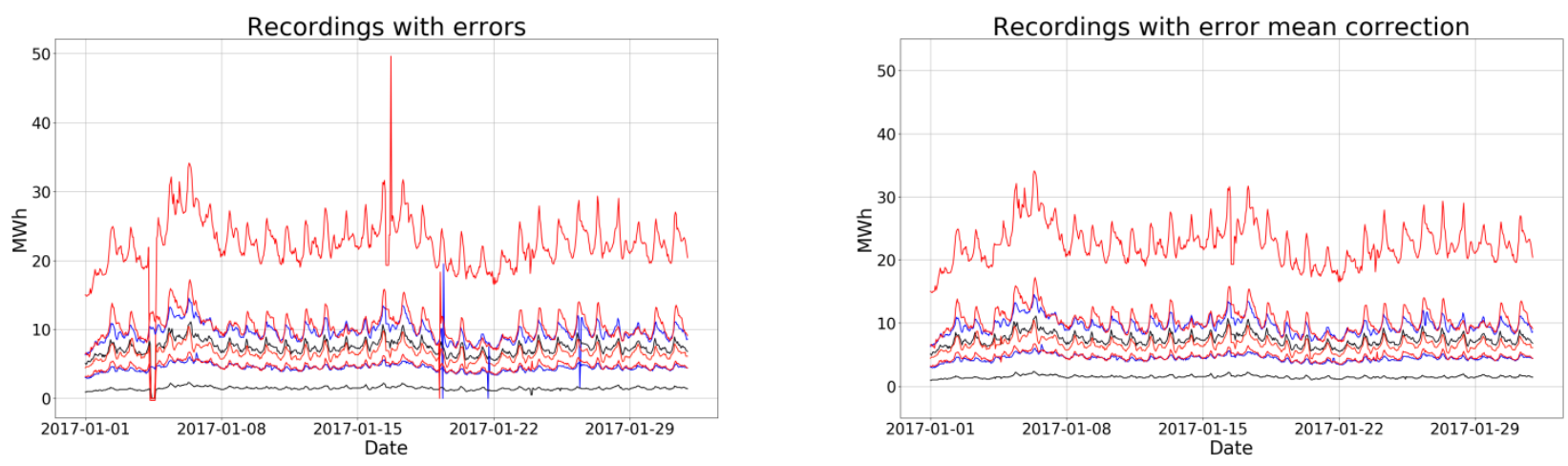

153

154

155

156

157

158

159

160

Figure 1 - (left) Original meter readings from HX stations with clear recording errors. Affected HX stations 111C, 119, 133, 134, 135, 136, 148 and 151. (Right) Correction of errors by imputing series mean into the faulty reading.

No other concerns were observed in the data; hence the error mean correction is the only data manipulation applied to the data before analysis, resulting in a final sample size of 49 meters each with 744 readings encompassing January 2017. A recent study analyzing district heat end user consumption investigates household smart meters from the same region whereas this study analyzes district heat exchange stations servicing larger areas [22]. 


\section{Methodology}

This section will outline the methods applied to the data. Starting with a description of the clustering technique K-Means in section 4.1, section 4.2 will introduce data scaling, which is needed for K-Means. Section 4.3 describes the selection of the number of clusters. Cross-validation is introduced in section 4.4. Finally section 4.5 and 4.6 introduces transformations of the input data by way of autocorrelation functions and wavelets.

\subsection{K-means Clustering}

Clustering HX stations into smaller homogenous subsets for better encapsulating consumption structures is an unsupervised problem. There is no prior knowledge of the true underlying clusters and hence no known structure to model against. In comparison, supervised classification utilizes a response variable, usually denoted $\mathrm{Y}$, and a link function to model the underlying structure. From previous studies of electricity smart meter clustering [2] a considerable amount of clustering is performed by applying K-means or derivatives of the K-means algorithm. Consequently K-means will act as a benchmark for the analysis performed in this paper. $\mathrm{K}$ means has some properties which make it a popular choice for unsupervised clustering, but there are some caveats which must be addressed.

K-means is readily implemented in most modern analysis software from proprietary to open source. In absence the algorithm is simple to implement. The algorithm is very robust and usually able to cluster data satisfactory. There exist K-means derivatives which are optimized for handling outliers or allowing fuzzy class-labels. The simplicity of the algorithm is its raison d'etre, in comparison to more advanced algorithms which do not necessarily perform significantly better. This further propels the popularity of general purpose algorithms like the K-means algorithm for unsupervised clustering.

There are some significant caveats concerning the K-means method, most significant is its greedy design philosophy [23] combined with the random initialization of the algorithm. The algorithm is prone to identify locally optimal solutions, which can lead to results that are not globally supported. To alleviate this problem the algorithm is usually run several times with random initialization and then selecting the best solution among the runs. This paper utilizes the K-Means implementation found in the scikit-learn framework for python [24] which by default selects the best clustering of 10 repetitions. Unless the initial random seed is fixed, the algorithm will return new clusters each run. Throughout this paper the random seed is set to 12345 to ensure reproducibility.

The algorithm does not account for any inherent structure in the data, for instance autocorrelation often found in time series. Either the algorithm is improved to handle more data structures or the input data can be preprocessed in such a fashion that the algorithm can cope with the inherent information in specific data structures. In this paper, we will pursue preprocessing of data to improve clustering performance.

The algorithm is initialized with k number of clusters; the clusters can be random or predefined. Repeatedly each observation is assigned the cluster which is closest to the observation, after which the cluster centers are recalculated including the newly assigned observation. This process is continued until no change occurs in 
cluster assignments and the algorithm has converged to at least a local optimum. For an intuitive and thorough discussion of the mechanics of the algorithm see $[25,26]$.

\subsection{Data Scaling and translation}

The K-means algorithm compares data by difference, grouping HX stations with large energy throughput together regardless of their consumption pattern. To avoid classifying the amount of energy consumed rather than the pattern by which it was consumed, we need to remove scale from the data. Similarly [11] applied scaling as preprocessing of smart meter data. In this paper we apply four different scaling and two transformations. The transformations are intended to amplify the difference between the groups, which makes it easier to recognize homogeneous clusters.

\begin{tabular}{|l|c|l|}
\hline Scale & Mathematical Description & Intuition \\
\hline Normalization & $\frac{x-x_{\text {min }}}{x_{\max }-x_{\min }}$ & $\begin{array}{l}\text { Normalization puts all observations } \\
\text { on a 0-1 scale compared to the } \\
\text { largest reading. Dimensionless. }\end{array}$ \\
\hline Standardization & $\frac{x-x_{\text {mean }}}{\sigma}$ & $\begin{array}{l}\text { Standardization scales all } \\
\text { observations compared to the } \\
\text { variance of the time series. } \\
\text { Dimensionless. }\end{array}$ \\
\hline Mean-Center & $x-x_{\text {mean }}$ & $\begin{array}{l}\text { Mean-centering removes the mean } \\
\text { from the meter reading. It is equal } \\
\text { to shifting on the y-axis. }\end{array}$ \\
\hline Mean-Divide & $\frac{x}{x_{\text {mean }}}$ & $\begin{array}{l}\text { Scales observations relative to the } \\
\text { series mean. Does not constrain } \\
\text { the y-axis to the interval [0, 1]. } \\
\text { Dimensionless. }\end{array}$ \\
\hline
\end{tabular}

Table 2 - Scaling methods applied in this paper, to remove consumption volume impact from HX stations. Scaling can help reveal true consumption patterns and disregard volume throughput differences of individual $\mathrm{HX}$ stations.

The 4 different scaling techniques listed in Table 2 all remove the differences in consumption volume while retaining the consumption patterns of each $\mathrm{HX}$ station. Normalization scales readings from each $\mathrm{HX}$ station to the interval [0:1]. Standardization scales the consumption patterns with respect to standard deviation of the $\mathrm{HX}$ station with mean equal 0 and unit variance. Mean-Centering scales by removing the mean from the HX station, shifting the mean to 0 . Mean-divide is comparable to normalization, dividing by series mean. It does not constrain the $y$-axis to the interval $[0,1]$.

The scaling methods remove volume differences, through different strategies, essentially retaining only the pattern in the data. Scaling can be essential in clustering techniques where clustering is performed on a distance metric. As mentioned $\mathrm{K}$-means is prone to cluster $\mathrm{HX}$ stations of equal consumption volume regardless of difference in consumption pattern. The data are scaled to ensure HX stations are clustered by pattern and not consumption volume. Figure 2 (top) shows the original data and the implications of the different scaling applied (bottom), which illustrate that removing consumption volume difference of the HX stations reveals similar consumption structure across the stations. 

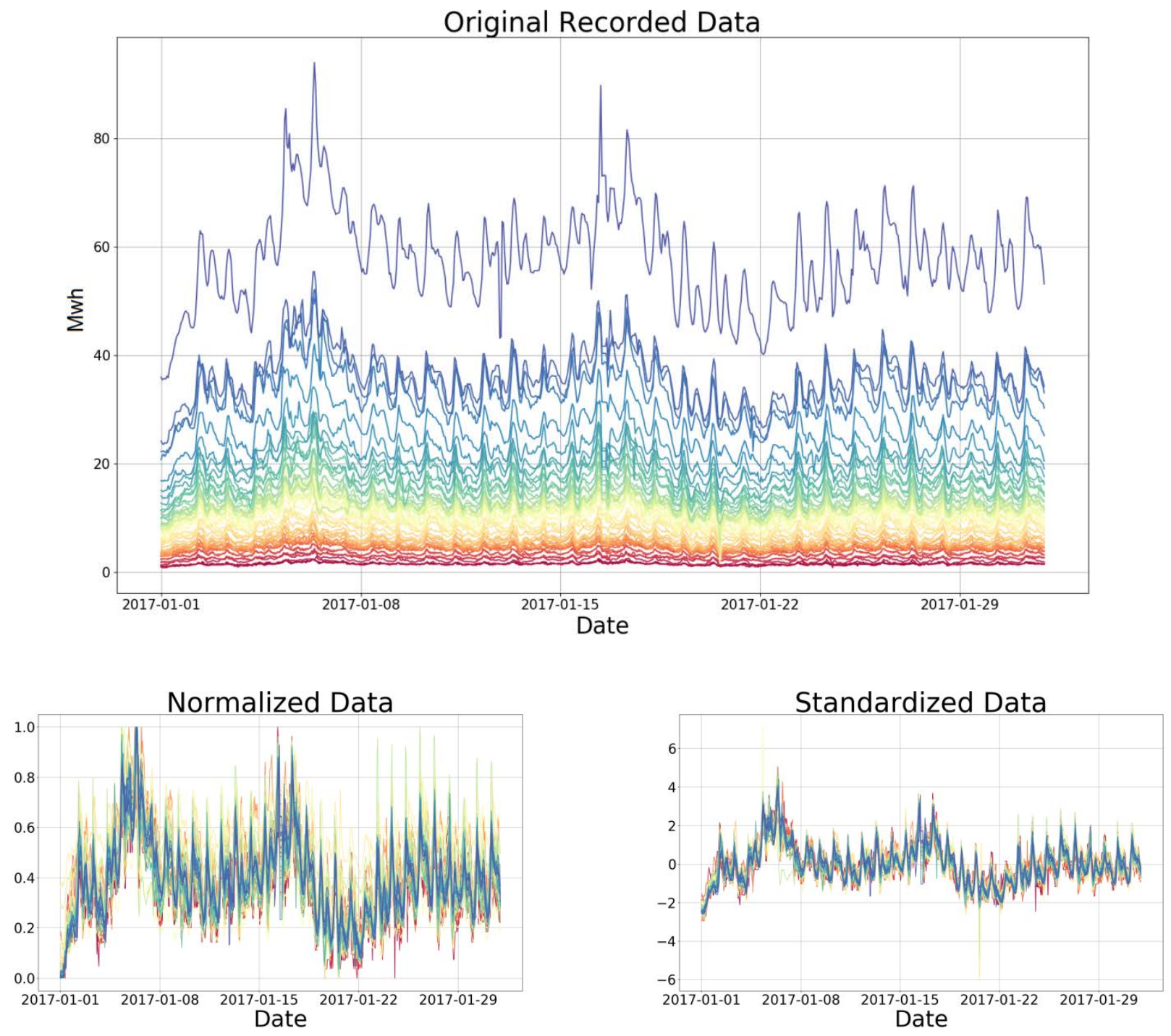

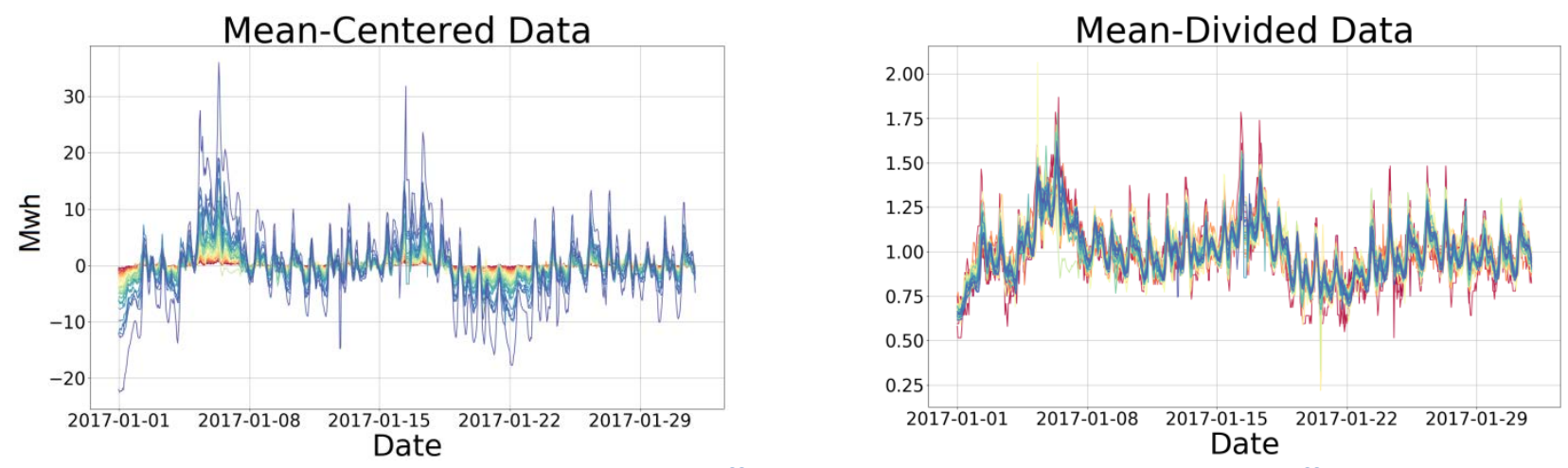

222

223

224

225

226

227

228

229

230

231

232

233

234

235

Figure 2 - Top: the original data plotted, clearly showing a difference in consumption volume rather than different consumption patterns. Mid-Left: normalizing data reveals the similar consumption pattern for each HX station. Likewise does standardization, mean-centering and mean-dividing of the data. The scaling reveal similar consumption pattern across all $49 \mathrm{HX}$ stations with different consumption volume. The coloring shows the small differences in the different HX stations consumption after scaling.

\subsection{Selecting the Number of Clusters}

K-Means does not give a solution to selecting the optimal number of clusters. It purely classifies according to the initial user-selected number of classes and the random seed. In order to select the optimal number of clusters, we need a metric for evaluating different clustering solutions, without knowing the true underlying clusters. A multitude of different measures, for assessing clustering performance have been developed to help identify the optimal number of clusters. These metrics are concerned with quantifying inter and intra variability of the resulting clusters. This paper will employ 4 different cluster validation indices: MIA, Cluster Dispersion Indicator, Davies-Bouldin Index and the Silhouette index. All indices applied are described in Table 3 which is an adaptation of a table presented in [2].

\begin{tabular}{|c|c|c|}
\hline Index & Mathematical description & Properties \\
\hline MIA & $M I A=\sqrt{\frac{1}{K} \sum_{k=1}^{K} d^{2}\left(C_{k}\right)}$ & $\begin{array}{l}\text { Average distance within class-member to class centroid, } \\
\text { summarized across all classes. } k \text { is number of clusters; } d^{2}\left(C_{k}\right) \\
\text { is the squared average distance within cluster } k \text {. High MIA } \\
\text { indicates large distances within the classes. E.g., large } \\
\text { dispersion. }\end{array}$ \\
\hline $\begin{array}{l}\text { Cluster Dispersion } \\
\text { Index (CDI) }\end{array}$ & $C D I=\frac{1}{d(C)} \sqrt{\frac{1}{K}} \sum_{k=1}^{K} d^{2}\left(C_{k}\right)$ & $\begin{array}{l}\text { CDI prefers Long inter-cluster distance and short intra- } \\
\text { cluster distance [14]. Small values indicate good clustering. } \\
d^{2}\left(C_{k}\right) \text { is the squared average distance within cluster } k \text {. While } \\
d(C) \text { is average cluster distance in data [14]. }\end{array}$ \\
\hline $\begin{array}{l}\text { Davies-Bouldin } \\
\text { Index (DBI) }\end{array}$ & $D B I=\frac{1}{K} \sum_{i=1}^{K} \max _{j \neq i} \frac{\operatorname{diam}\left(C_{i}\right)+\operatorname{diam}\left(C_{j}\right)}{d\left(C_{i}, C_{j}\right)}$ & $\begin{array}{l}\operatorname{diam}\left(C_{k}\right) \text { is the average diameter of a cluster. And } d\left(C_{i}, C_{j}\right) \text { is } \\
\text { the distance between cluster centers. } K \text { is the number of } \\
\text { clusters. DBI relates the mean distance of each class with the } \\
\text { distance to the closest class [27]. Smaller values of DBI } \\
\text { implies that K-means clustering algorithm separates the data } \\
\text { set properly [16] }\end{array}$ \\
\hline Silhouette Index & $\begin{aligned} \text { Silhouette } & =\frac{c^{\prime}(x)-c(x)}{\max \left\{c(x), c^{\prime}(x)\right\}} \\
c^{\prime}(x) & =\min _{y \in C^{\prime}} d(x, y)\end{aligned}$ & $\begin{array}{l}c(x) \text { is the average distance between vector } x \text { and all other } \\
\text { vectors of the cluster } c \text { to which } \mathrm{x} \text { belongs. } c^{\prime}(x) \text { is the } \\
\text { minimum distance between vector } \mathrm{x} \text { and all other vectors in } \\
\text { cluster } \forall C^{\prime} \neq C[11] \text {. } \\
\text { SI is between }[-1,1] \text { higher is better. Negative is miss- }\end{array}$ \\
\hline
\end{tabular}


Table 3 - Overview table of Cluster validation indices applied in this paper, with their mathematical description and intuitive properties. The table is an adaptation from a table in [2].

Like residuals and $r^{2}$ in regression analysis, these indices are developed so as to minimize the dispersion within and maximize the distance between clusters, helping to select the optimal number of clusters. Plotting the progression of the indices as function of clusters allows for visual inspection, where abrupt changes in their decline or fluctuating pattern can help select the number of clusters within a given data set. This method of estimating the number of clusters in unsupervised clusters has been studied by [28].

\subsection{Cross-Validation}

Cross-validation is a concept developed for supervised learning [29] as a bias-variance trade-off for reducing misclassification by splitting the data into a test and training set [30]. In the test set the true cluster label is known, which enables comparison of the model clustering vs the true clusters.

We will apply cross-validation, not directly to the unknown clusters but to the cluster-validation indices thus regarding the indices as pseudo cluster labels. We apply leave-one-out cross-validation [31], calculating the indices for all $49 \mathrm{HX}$ stations with the omission of one. This is done repeatedly until indices have been calculated for each combination of $48 \mathrm{HX}$ stations. We report the maximum, minimum and average index value, of all four validation indices.

\subsection{Autocorrelation Feature Extraction}

The HX station meter readings are ordered in time, with equidistant intervals, each HX stations data output can be regardeds as a time series. An extensive literature exists on Time Series Analysis which we will not cover in this paper. For a thorough discussion refer to [32], for a survey on time series clustering with discussion on difference in type of methods see [33]. An important tool from classical Time Series Analysis is the autocorrelation function (ACF) which is defined as:

$$
A C F(\tau)=\frac{E\left(\left[X_{t}-\mu\right]\left[X_{t+\tau}-\mu\right]\right)}{\sigma^{2}}
$$

where $t$ and $\tau$ are integer time steps, $\mu$ is the series mean, and $\sigma^{2}$ it the series variance. Intuitively the data generating process is correlated with itself, quantifying how much previous observations influence the current observation. The $\mathrm{n}$ lags in an ACF plot indicate the influence of each of the previous $\mathrm{n}$ observations and they are essential in identifying the type of time series process which generated the readings. The ACF plot visualizes inherent information such as dampening, oscillation, and recurrences throughout the data. The data are expected to contain autocorrelation as the output from the HX stations is expected to exhibit a daily cycle. To the best of our knowledge this has only briefly been studied in smart meter electricity analytics by [20] and has not been utilized as input for K-Means clustering in a smart meter setting. In this paper we will not perform a rigid time series analysis of each HX station, nor will we develop ARIMA models for the individual HX Stations. 
As we do not develop ARIMA models we do not need to ensure stationarity of the time series. We will merely evaluate differences in ACF and apply the coefficients as input for the K-Means algorithm to cluster.

$\mathrm{K}$-means is by default not leveraging the expected auto-correlation information in the HX station readings as mentioned in section 4.1. We preprocess the input data by calculating the ACF coefficients of each HX station, and input the resulting ACF coefficient matrix into the K-Means algorithm thereby enabling the K-Means to account for the autocorrelation in the data. K-Means is enabled to account for auto-correlation by classifying the ACF structure rather than the observed consumption data from each station. As the ACF is employed to characterize the underlying model and parameters, clustering the ACF coefficients can be regarded as clustering with respect to the underlying process. Under certain conditions a rigid time series analysis and model can be developed describing the individual clusters.

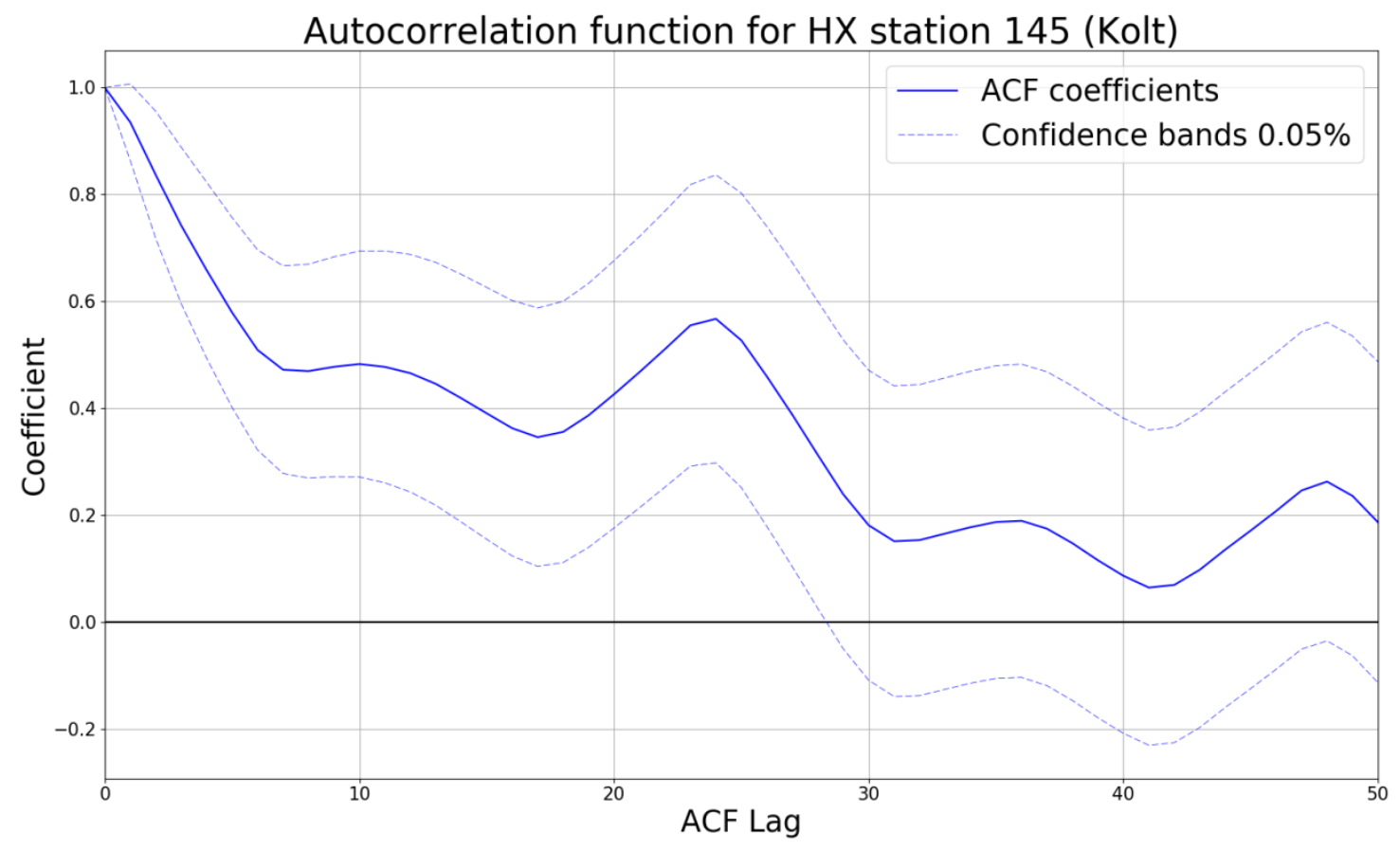

Figure 3 - Autocorrelation plot with 50 lags of HX station 145 the town of Kolt. The $0.95 \%$ confidence bands shows significant lag coefficients until lag 28. A clear seasonality is also seen at lag 24 indicating a daily recurrent pattern.

Figure 3 shows a 50-lag autocorrelation plot including 95\% confidence intervals of the HX station; 145 Kolt. The Confidence intervals shows the first 28 lag coefficients to be significantly different from 0 . The Figure confirms the existence of autocorrelation in the data. Moreover the Figure shows a seasonality component every 24 lag, indicating a recurrent daily cycle. The ACF is invariant to the scaling and translation described in section 4.2 meaning volume differences are irrelevant when ACF preprocessing the data. 


\subsection{Wavelet Feature Extraction}

The wavelet transformation is a basis transformation using wavelet basis functions. Wavelets are able to represent smooth and locally non-smooth functions. Wavelets have time and frequency localization, effectively linking time and frequency in contrast to the Fourier transformation which only allows frequency localization [31]. Wavelet are especially well suited for analyzing high frequency data because of their ability to capture global smoothness and local spikes in the signal [30], while filtering out high frequency noise [34]. The application of wavelets for time series feature extraction in this study has been inspired by [35]. In the process of filtering high frequency data, wavelets perform efficient data compression, by removing non-significant coefficients. Often this process removes a considerable number of coefficients. The decomposition of the signal into wavelet coefficients are not easily human interpretable, but are readily applicable as input for the KMeans algorithm. The wavelet coefficients are uncorrelated [36].

299

300

301

302

303

304

305

306

307

308

A wavelet defines the orthonormal basis function, and is scaled and shifted to fit the signal. Choosing a suitable wavelet can be difficult, as the scaled basis wavelet must be able to encapsulate the structure of the signal. For the selection of the wavelet applied for analysis of the HX data refer to section 5.3. As wavelet definitions are often very complex, we here present a brief overview of the theory of wavelets using the Haar wavelet. The Haar wavelet is defined by [37]:

$$
\psi(x)=\left\{\begin{array}{r}
1, \text { if } 0 \leq x<\frac{1}{2} \\
-1, \text { if } \frac{1}{2} \leq x<1 \\
0 \text { Otherwise }
\end{array}\right.
$$

and is shown in Figure 4. The basis function is scaled and shifted by the scaling function defined as:

$$
\psi_{j, k}(x)=2^{j} / 2 \psi\left(2^{j} x-k\right)
$$

where $\mathrm{j}$ and $\mathrm{k}$ are integers. This function has the same shape as $\psi$ but is scaled and shifted [30]. The wavelet is repeatedly applied at different scales to locally fit any fluctuating and smooth regions.

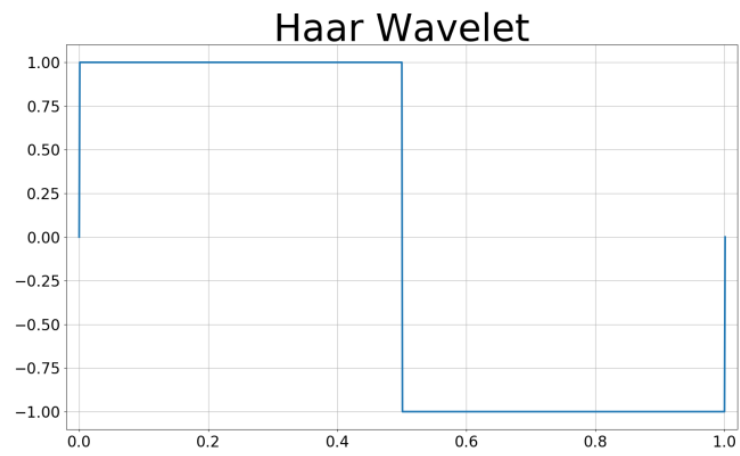



$D_{j, k}$ which are kept as wavelets parameters $\hat{\beta}_{j, k}$. The coefficient is evaluated for statistical significance by:

$$
\hat{\beta}_{j, k}= \begin{cases}D_{j, k} & \text { if }\left|D_{j, k}\right|>\hat{\sigma} \sqrt{\frac{2 * \log (n)}{n}} \\ 0 & \text { othervise }\end{cases}
$$

Wavelets other than the Haar wavelet are usually difficult to describe in closed form, but numeric tools for computation are available[30]. There exists a fast algorithm for wavelet coefficient calculation which processes in linear time [38], which in algorithmic analysis o-notation is equivalent to $O(n)$ [23]. The python wavelet package PyWt [39] was utilized for the wavelet analysis presented in this paper. Figure 5 (Left) shows a Haar wavelet transformation fit to the HX station; 145 Kolt. The original time series has been transformed via the wavelet, retaining only significant coefficients. The wavelet transformation has compressed the time series to 161 coefficients compared to the original data of 744 observations. The figure shows how the wavelets are able to keep the important structure in the data even at a 1:5 compression. The structure of the Haar wavelet results in its inability to truly follow the data, producing noise reduced representation of the original data. The corresponding Haar wavelet pyramid plot of $\mathrm{HX}$ station 145 Kolt is seen in Figure 5 (Right).

HX Station 145 Kolt vs Wavelet Recovery

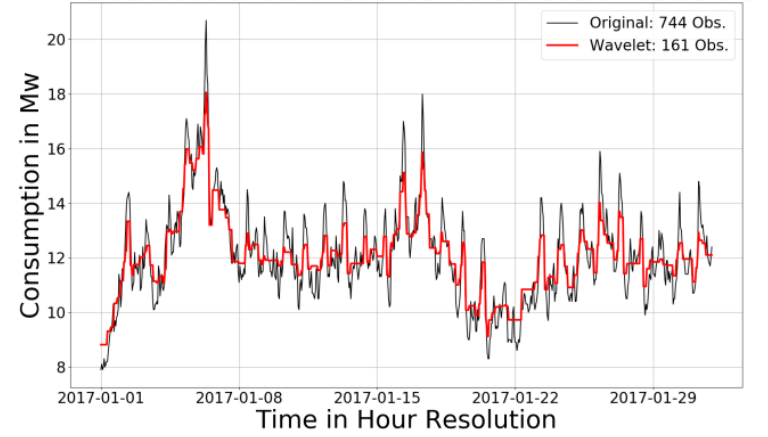

Haar Wavelet Coefficients for HX: 145 Kolt

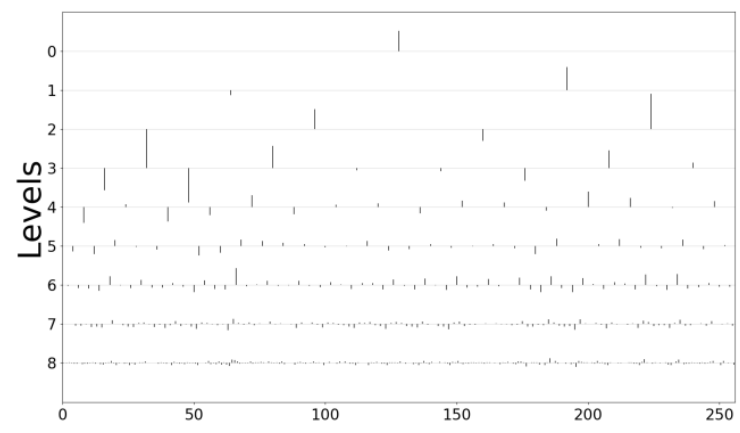

Figure 5 - (Left) Illustration of Haar wavelet approximation to original data series. The wavelet has a compression factor close to 1:5 and still recovers large parts of the structure in the original time series. Only 161 wavelet coefficients were significant different from zero, compared to the entire data series of $\mathbf{7 4 4}$ observations. (Right) Haar wavelet pyramid plot after applying Haar wavelet to the HX station 145 Kolt.

The visual analysis of wavelets is done using the pyramid plot. The algorithm compares neighboring observations pairwise e.g. in the small time series $x=[1,2,3,4]$ observation $(1,2)$ are compared, and $(3,4)$ are compared. This comparison yields the first level in the pyramid algorithm. Next pair $(1,2)$ and pair $(3,4)$ are compared, producing the second level, and so forth until there are no more pairs to compare. This can be plotted as a pyramid-plot with the lines indicating size of the difference from the comparison done at each level. Universal thresholding is then applied on the lowest level to remove noise. For clustering we generate new data series from the coefficients and cluster the HX stations by their wavelet coefficients. 
This section will present the results from K-Means clustering performed on scaled data in section 5.1, while section 5.2 compares clustering performance on autocorrelation transformed data, and subsequently in section 5.3 the results from the wavelet transformation is presented. Section 5.4 presents a comparison of the different clustering achieved

\subsection{Cluster Performance: Normalized Data}

We see how well we can classify HX stations with K-Means and whether careful transformation of the original data can improve the performance by applying scaling and ACF transformation to the input data. K-Means clustering done on scaled data constitutes our benchmark for comparing the input transformation influence on 343 K-means clustering performance.

344 To the best of the authors' knowledge HX stations have never before been classified by applying smart meter 345 recordings. Our hypothesis is that the data will behave similarly to electricity smart meter data, we therefore 346 progress with the analysis of $\mathrm{HX}$ stations in identical manner as observed in [2] regarding electricity smart 347 meter data.

348 We plot the cluster validation index to select the optimum number of clusters for the scaled data and study 349 how they develop as the number of clusters increases from 2 to 20 clusters. All 4 validation indices; Silhouette, $350 \mathrm{MIA}, \mathrm{CDI}$ and DBI are calculated for each of the scaling, Normalized, Standardized, Mean-Centered, and Mean351 Divided. Figure 6 shows how the mean of 4 indices develops as more clusters are introduced. The dark grey box 352 overlay indicates a potential optimum number of clusters.
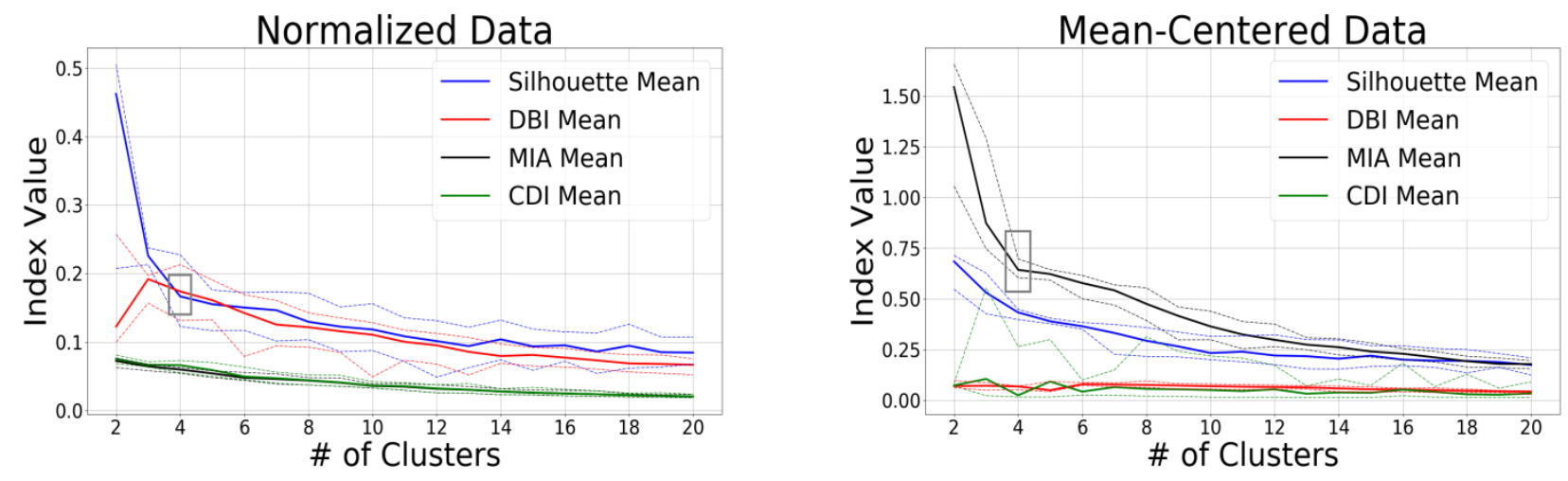

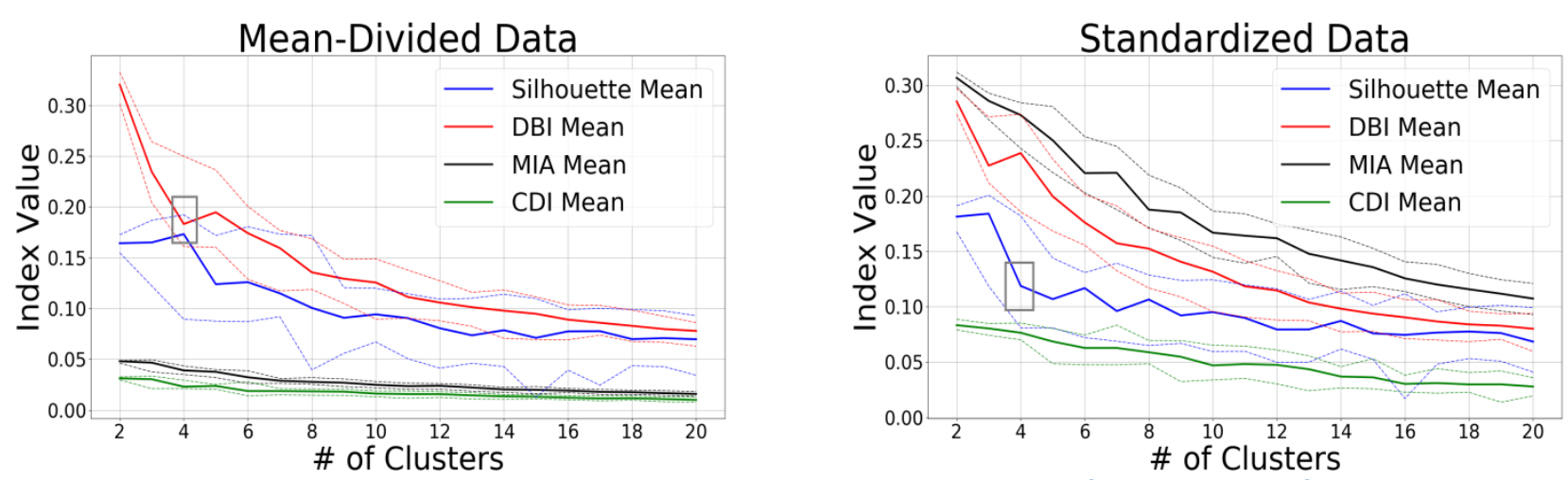

353

354

355

356

357

358

359

360

361

362

363

364

365

366

367

368

369

370

371

372

373

374

375

376

Figure 6 - Cluster number selection. Random Seed: 12345. Pseudo Cross-validation is performed on each of the validation indices. The maximum and minimum recorded value for each index and cluster number is shown with dashed lines, while the bold line is the average index value. The dark grey box indicates the optimum number of clusters for each scaling.

The silhouette index performs especially well for the normalized data, with an "elbow" break at four clusters indicating marginal performance increase by including more clusters. For the mean-centered data the MIA index shows an "elbow" break around four clusters. For the Mean-divided data the DBI indicate an "elbow" break occurs at four clusters, while the silhouette index is close to the DBI index. Finally the Standardized data shows a steady almost linear decline of the indices indicating no apparent cluster size cutoff. The CDI index is not indicating any number of clusters for any scaling following a linear decline in each case. Three different indices indicate four as the optimum number of clusters, which will be our choice of clusters.

It is not apparent from Figure 6 which of the scalings to apply. Only standardization falls short of giving an indication of how many clusters to include. We select normalization with four clusters as it yields the most balanced clusters where the smallest cluster has four members. Selecting balanced clusters reduce the risk of clustering outliers. Unbalanced clusters such as the mean-divided and the mean-centered in Table 4 have clusters with only one member this is comparable to an outlier having its own cluster. Clustering outliers in this setting is undesirable as we want to identify groups of similar consumers, Table 4 shows the cluster size after scaling.

\begin{tabular}{|l|l|}
\hline Transform & Cluster size @ 4 clusters \\
\hline Normalization & $(18,4,12,15)$ \\
\hline Mean Divided & $(5,29,14,1)$ \\
\hline Mean Centered & $(21,4,23,1)$ \\
\hline Standardization & $(11,12,22,4)$ \\
\hline
\end{tabular}

Table 4- Resulting cluster sizes with 4 clusters and transformations; Normalized, Mean-Centered, Mean-Divided and standardization. Normalization yields the most balanced clsuters.

The resulting clustering; four clusters and normalized data, is shown in Figure 7 (left) where the 4 cluster means are plottet. The means exhibit the same general structure with a slight offset and it is possible to destinguish individual cluster means. Superimposing the individual cluster members onto the graph Figure 7 (right) visualizes the overlap between the classes. The clusters are seperated but not to such an extend that they are easily separable due to large within cluster variation resulting in overlapping clusters. 

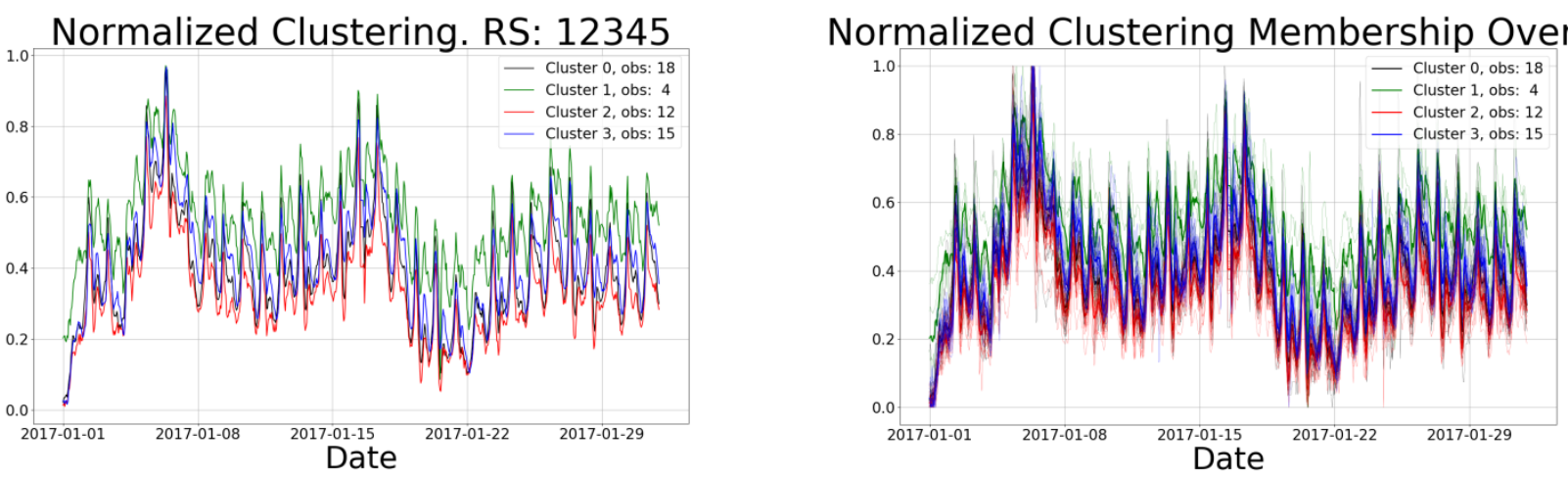

Figure 7 - (Left) 4 class means, after normalization. (Right) overlay of all class member onto the class means, cluster blue, red and black are very close while the green cluster is distingushable form the other 3 clusters.

379 This effectively renders the clustering an academic exercise that shows K-means can be applied to successfully identify clusters, but their true separability and practical applicability is questionable. The overlap of the clusters was expected due to the low index values seen in Figure 6, especially the CDI indicated clusters could be overlapping. The outcome does not change by changing the meter window from one month to weekly or daily basis which also results in overlapping classes.

The resulting clusters are closely located, which makes the individual clusters difficult to apply in a nonacademic setting. We have to be more aware of the features of the input data to the K-Means algorithm to remedy the situation. Clever data transformation is needed before clustering to circumvent the weaknesses of the K-Means. One of these methods could be the application of the autocorrelation structure as input features, enabling the K-Means to treat auto-correlated data when clustering. Figure 8 shows how the clusters are distributed across the consumption volume and not grouped with equal consumption clusters. 


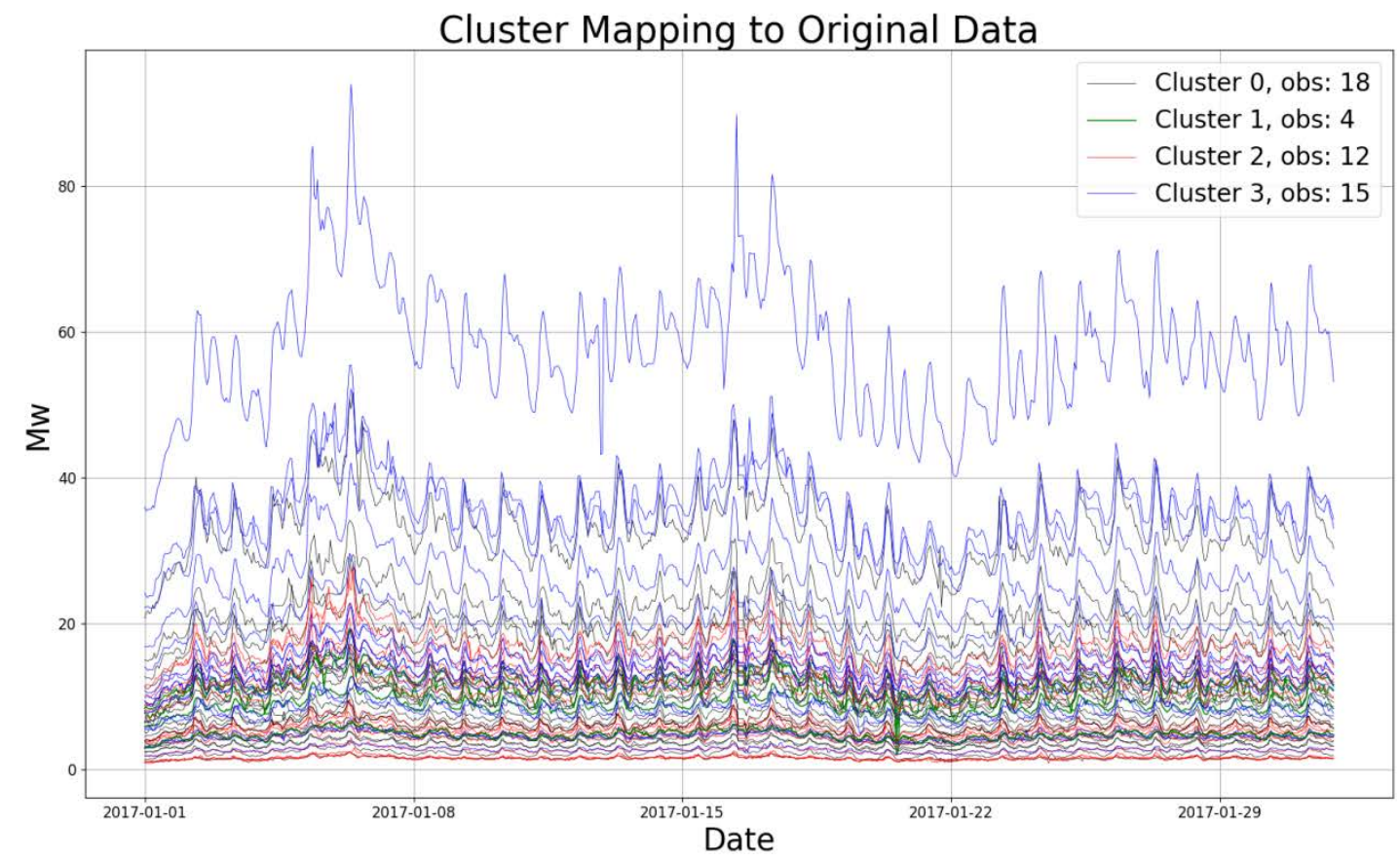

390

Figure 8 - Mapping of class members to original data. It is clearly seen that the clusters are not biased by consumption volume.

392

393

394

395

396

397

398

399

400

401

402

403

404

405

406

407

408

409

Clustering by applying scaled data ensures the clusters are not biased by differences in consumption volume of individual HX stations. Scaling alone does not utilize latent information inherent in the data. Contrarily, the ACF is invariant to the volume and focuses only on the underlying consumption structure of each HX station which makes scaling of the data irrelevant. Additionally the ACF also cater important information for Box-Jenkins classical analysis of time series and thus points the way for deeper knowledge of the underlying process that generated the consumption in each cluster through further analysis.

\subsection{Cluster Performance: Autocorrelation Feature Extraction}

To enable K-Means to account for autocorrelation in the data, we calculate the autocorrelation coefficients of all HX stations with 24 hours lag, and apply this as input to K-Means. Only significant autocorrelation coefficients are included in the input data set to ensure statistical stability. 24 hour lag has been chosen as the basis for the general structure encompassing one daily cycle. Figure 3 shows that coefficients above 24 lags become non-significant, with the recurrent pattern seen in the figure continuing for multiple lag beyond 24 lags. This pattern of significance was observed for all the HX stations.

The exact same method as described for clustering of scaled data has been applied to develop the 4 cluster validation indices as a function of number of clusters from 2 to 20 illustrated in Figure 9 (Left). There is a clear change in progression near 7 clusters. Figure 9 (right) shows the different cluster means for the 7 clusters, though the $7^{\text {th }}$ cluster only has two observations it is plotted for completeness. The resulting 6 clusters show visible differences and are more separable than the scaled clusters seen in Figure 7 (left). The separability of 
410 clusters is especially noticeable in the region lag 8 to lag 17, while at the end points the clusters exhibit similar 411 structure, Figure 9 (Right). Figure 9 (right) clearly shows a difference in the ACF for each cluster, the ACFs 412 indicate that clusters 2, 3, 4 and 5 exhibit similar model structure only off-set by differences in model 413 parameters, while clusters 0 and 1 are distinctively different.
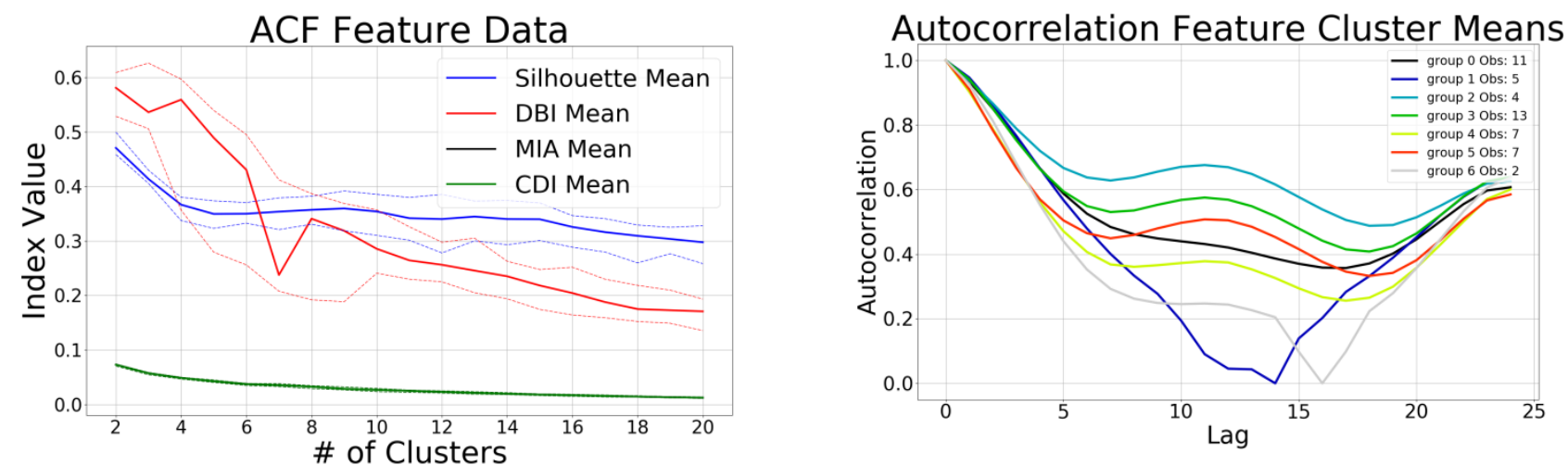

414 Figure 9 - (Left) Cluster validation index development, pseudo cross-validated, indicates a change at 7 clusters by the DBI index. The bold line is average index value while the dashed lines indicate minimum and maximum observed value for each index. (Right)

416 Cluster means from each of the 6 cluster which have 4 or more members.

417 The cluster membership overlay in Figure 10 illustrates how the dispersion of the different classes has

418 significantly reduced the overlap between clusters in the region lag 8 to lag 17. The clustering of ACF features 419 result in clearly separable clusters. 


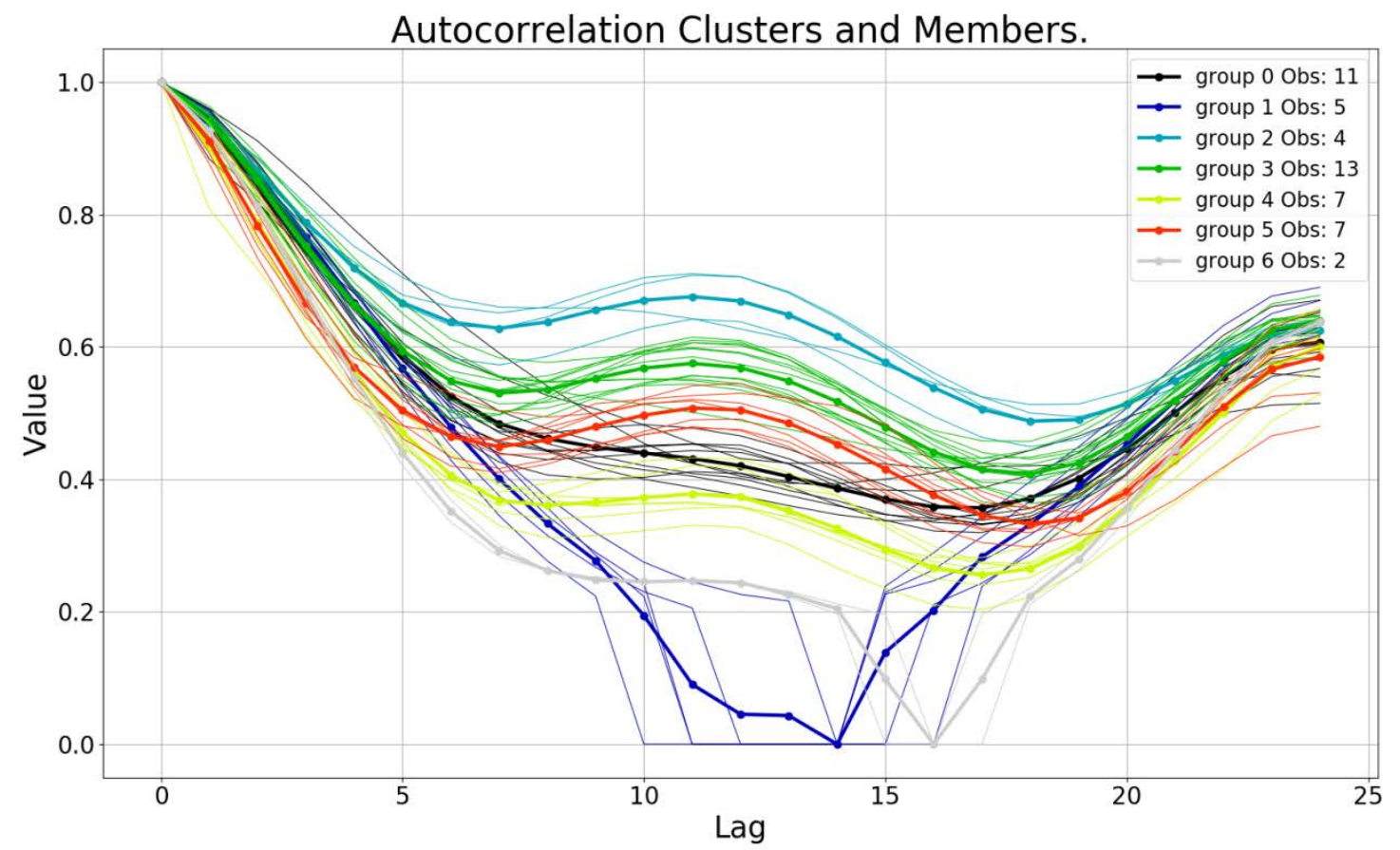

420

421 Figure 10 - Cluster member overlay. Colors represent cluster membership, and dots indicate cluster mean. It is clearly seen that the 422 cluster dispersion and subsequently overlap between different clusters is very small in the region lag 8 to lag 17 , yielding a better 423 discriminatory power.

424 There are more clusters identified with the ACF clustering compared to scaled clustering, the resulting ACF 425 clusters could potentially be sub-clusters of the scaled clusters. Table 5 shows this is not the case as the 7 ACF 426 clusters are scattered throughout the 4 scaled clusters. This clearly indicates a difference in the resulting 427 clustering by the 2 approaches.

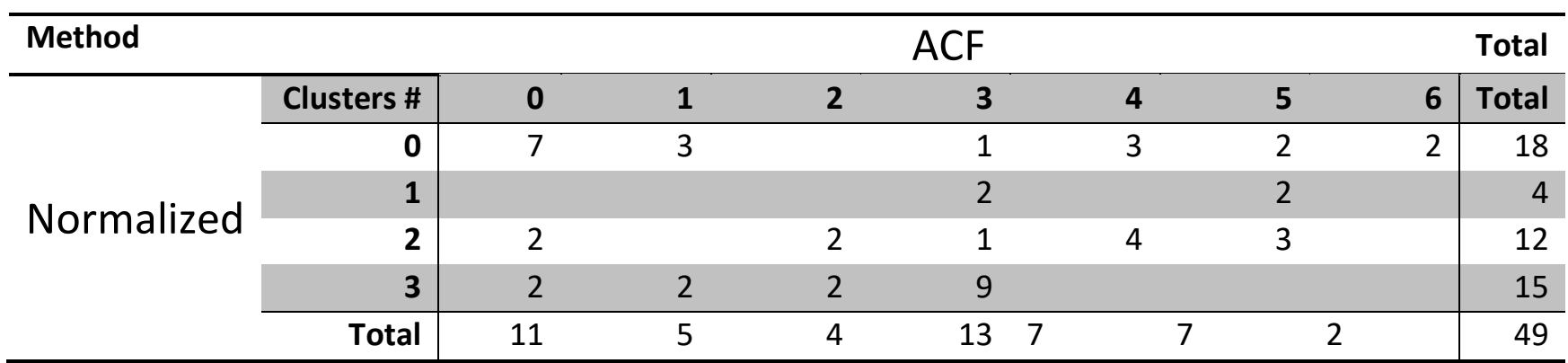

Table 5 - Cluster overlap table. Columns show the 7 clusters from the ACF transformation clustering, while rows show clustering with the Normalized. All Normalized clusters are scattered across several ACF clusters which shows that the detailed ACF clustering is not just a subset of the normalized clustering, but are entirely different clusters. 


\subsection{Cluster Performance: Wavelet Feature Extraction}

It is no trivial task to select the wavelet best suited to fit a given signal. The collection of documented wavelet basis function is extensive. Only wavelets already implemented in the PyWt python package has been evaluated to keep the task feasible in this study. This was done by fitting each wavelet family to every $\mathrm{HX}$ station, and selecting the wavelet with best overall fit on all HX stations. The Coiflet 16 wavelet shown in Figure 11 had the best overall fit. The wavelet transformation of the original data was done by retaining only significant wavelet coefficients and creates a wavelet coefficients input data set for the K-Means.

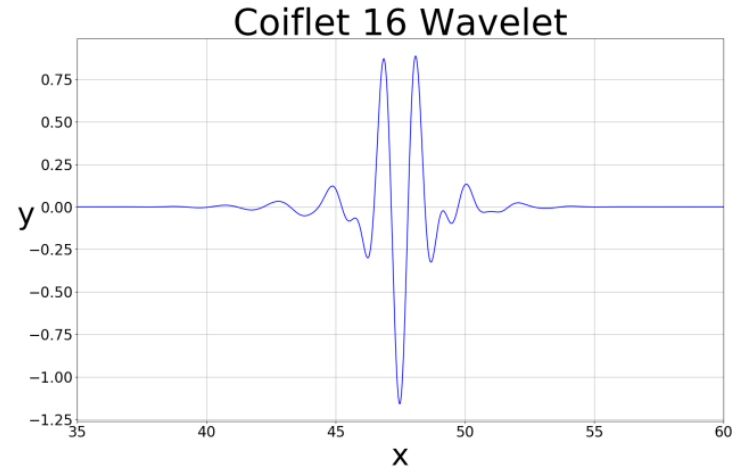

Figure 11 - Coiflet 16 mother wavelet selected, through testing, as the best wavelet for transformation of the smart meter data.

440 Finally we generate the cluster validation index progression shown in Figure 12 by applying the exact same procedure for estimating number of clusters as previously shown. All scaling, except mean-divided, indicate 4 clusters for the data. Mean-divided shows 4 or 5 clusters could be relevant. Normalizing and standardizing result in the most balanced clusters, but the index development for normalizing is more abrupt than for standardizing. All but the mean-divided scaling indicate 4 clusters. Even the original unscaled meter data suggests 4 clusters after wavelet transform. The 5 cluster solution for mean-divided results in very unbalanced clusters and is disregarded. We therefore conduct clustering on normalized data to ensure scaling is not an influencing factor to the wavelet coefficients for the remainder of the wavelet transform analysis.
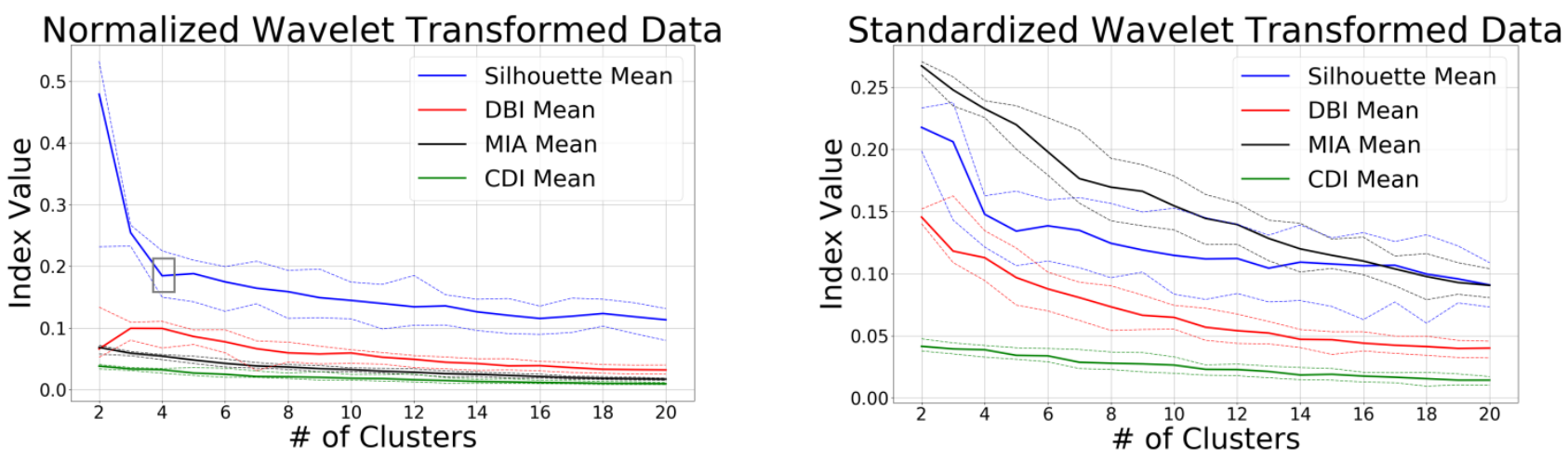
Mean-Centered Wavelet Transformed Data

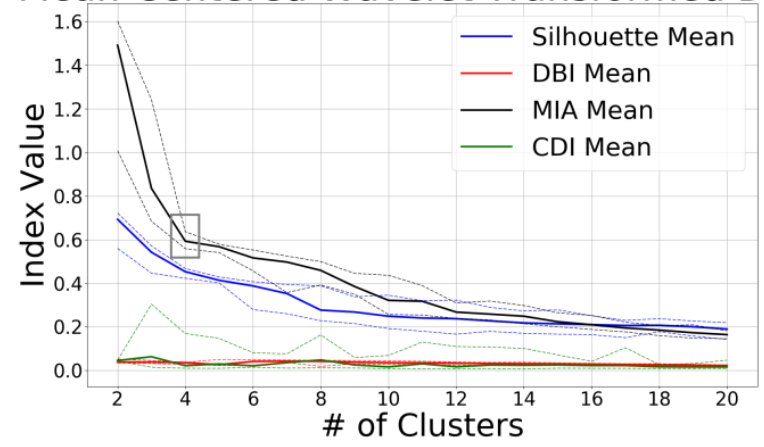

Mean-Divided Wavelet Transformed Data

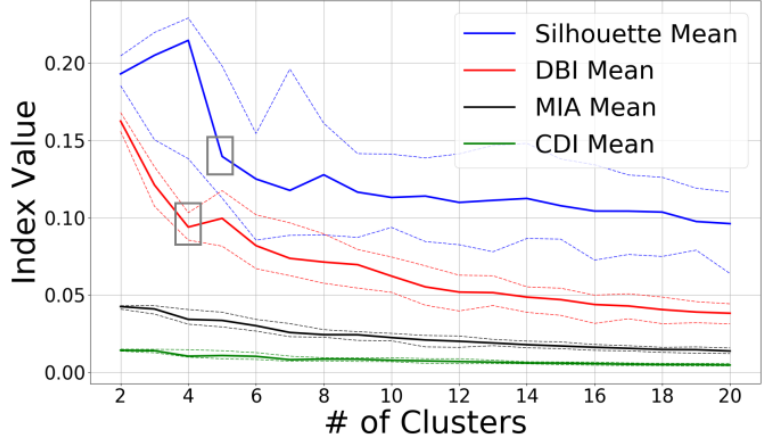

\section{Original Wavelet Transformed Data}

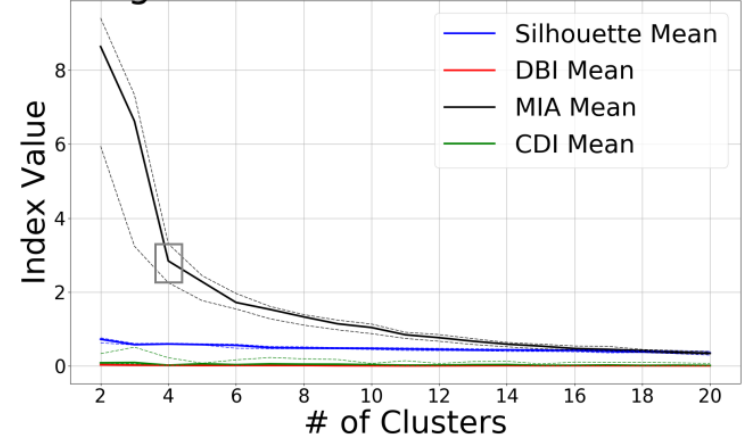

Figure 12 - All initial scaling, except mean-divided indicate same number of clusters for the wavelet transformed data. Coincidentally the same number of clusters suggested by the normalization scaling applied in $\mathbf{5 . 1}$. Mean-divided suggest 5 clusters which are more unbalanced and thus disregarded.

The resulting normalized cluster balance is $(20,4,9,16)$ which yield clusters with at least 4 members. Additionally, normalized scaling makes the results comparable to the clustering in section 5.1. The clustering using wavelet coefficients is shown in Figure 13. The plots are significantly different from the ACF and Normalized clustering due to how the coefficients are structured in the input data. Coefficients to the far right are from the lowest level of the pyramid and many are set to zero. From the figure it can be seen that the black clusters are shadowed by the red and green clusters, which was also observed with scaled clustering.
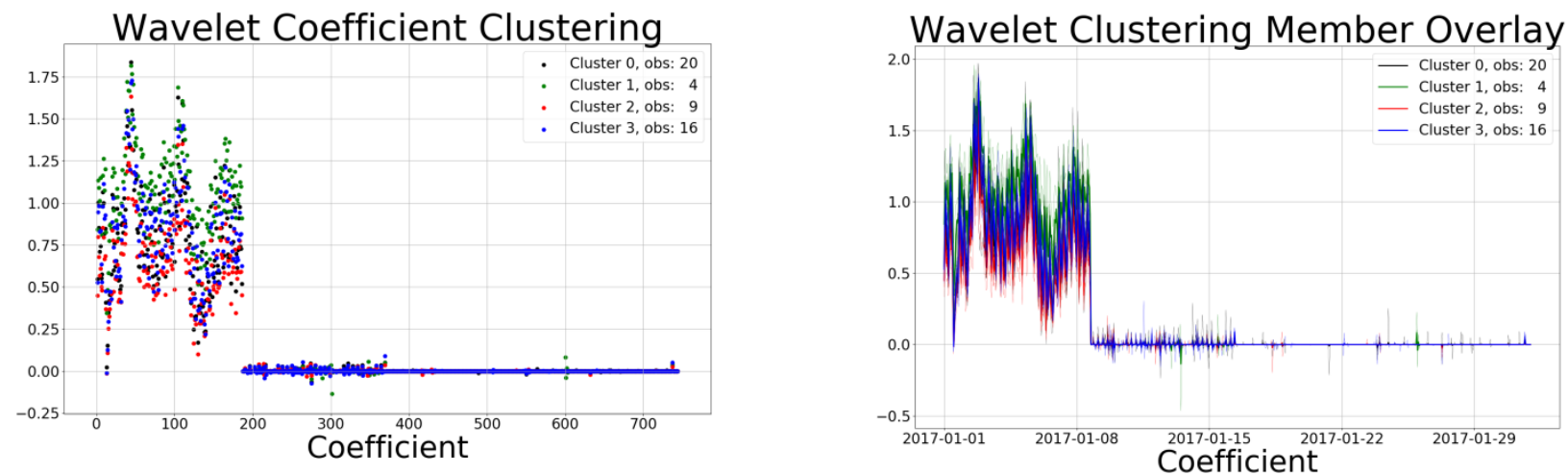

Figure 13 - (Left) Wavelet coefficient clustering. From left to right coefficients are from decreasing level of the pyramid algorithm. The 
The resulting clustering overlap between Normalized and wavelet clustering is shown in Table 6 . The clustering is very similar with only 6 of the $49 \mathrm{HX}$ stations clustered differently between the two methods.

\begin{tabular}{|c|c|c|c|c|c|c|}
\hline Method & & \multicolumn{4}{|c|}{ Wavelet } & \\
\hline & Cluster \# & 0 & 1 & 2 & 3 & Total \\
\hline \multirow[t]{5}{*}{ Normalized } & 0 & 16 & & & 2 & 18 \\
\hline & 1 & & 4 & & & 4 \\
\hline & 2 & 3 & & 9 & & 12 \\
\hline & 3 & 1 & & & 14 & 15 \\
\hline & Total & 20 & 4 & 9 & 16 & 49 \\
\hline
\end{tabular}

Table 6 - Clustering overlap table between Normalized and wavelet transformed data. Columns show wavelet transformed clustering and rows show Normalized clustering. Normalized and Wavelets produce similar clusters with this data set. Nearly all Normalized clusters are mapped 1:1 to the corresponding Wavelet cluster. The two methods yield similar clustering in this case.

461

462

463

464

465

466

467

468

469

470

471

472

473

474

475

476

477

478

479

480

481

482

483

484

485

486

\subsection{Comparison of Clustering}

The different clustering performed in sections 5.1, 5.2 and 5.3 have all utilized validation indices for selecting the number of clusters in the data, with "unknown ground truth" [33]. The three different methods applied in this paper results in different clustering solutions, though Normalization and wavelet transformation agree on 43 of the $49 \mathrm{HX}$ stations in 4 different clusters. ACF finds 7 different clusters which are not a subset of the Normalization clustering but entirely different clusters. All three methods result in statistical significant groups at $5 \%$ confidence level, meaning that overall the clustering in all three cases result in some clusters that are statistically distinguishable. The statistical testing conducted was analysis of variance [40] implemented in the python package Scipy [41]. Caveat to this is that all three clustering result also produced shadow clusters, clusters that shadow other clusters resulting in clusters that statistically are impossible to distinguish. This phenomenon was observed in all three cases, but ACF had fewer shadow clusters than Normalization and Wavelets.

While we have shown the transformation of the input data enables K-Means to handle autocorrelated data, we are unable to show statistical difference between the three cases. Another measure for evaluating the clustering is analyzing the computational effort needed to perform the clustering. All three cases preprocess the input data, in processes that can be run in linear time. The transformations influence on the overall runtime is negligible compared to K-Means lower bound runtime of $2^{\sqrt{n}}$ [42] and upper bound of $O\left(\mathrm{k}^{\mathrm{n}}\right)$ [42], where $k$ is clusters and $n$ is observations. Though the autocorrelation and wavelet transformations are efficient, it is their ability to reduce the number of dimensions of the input data which contributes most to the increase in overall running time. The transformations significantly reduce the number of dimensions and thus the worstcase running time for the clustering. At the same time the transformations enable K-Means to manage temporal components in the data. The inclusion of temporal components in the K-Means clustering allows for the clustering to account for intrinsic information about repeatability in consumption which is not modeled when the temporal component is ignored. This produces clusters that incorporate more information than without autocorrelation feature or wavelet features. 
This reduction of the input data via the wavelet or ACF feature extraction decrease the dimensions from 744 to 161 or 24 coefficients respectively and results in a dramatic decrease in minimum and worst-case computational effort needed to cluster the data, Table 7. The linear time consumption of the transformations are negliable compared to the computations needed for the K-Means clustering, thus the preprocessing of the data results in overall better runtime performance as seen in Table 7.

\begin{tabular}{|l|c|c|c|}
\hline & Scaled & ACF & Wavelet \\
\hline Scaling / Transform & $\mathrm{O}(\mathrm{n})$ & $\mathrm{O}(\mathrm{n})$ & $\mathrm{O}(\mathrm{n})$ \\
\hline Size of input data $(\mathrm{n})$ & 744 & 24 & 161 \\
\hline Best case running time & $2^{\sqrt{744}}$ & $2^{\sqrt{24}}$ & $2^{\sqrt{161}}$ \\
\hline Worst case running time & $4^{744}$ & $7^{24}$ & $4^{161}$ \\
\hline
\end{tabular}

Table 7 - Clustering method comparison. The different scaling and transformations presented are able to identify clusters in the data. Selecting one instead of another is difficult. In this case we can see the worst-case running time for the ACF clustering is better than the scaled or wavelet transformed data.

This paper has applied learnings from smart meter electricity consumption clustering to district heat exchange station clustering. Clustering of aggregated consumption data can aid the district heat utilities with optimizing heat production in their current grid and reduce capital expenditures when expanding their supply area and grid. The aim has been to investigate if the same methods are readily applicable to district heat clustering. The data utilized in this paper is generated by smart meters installed at Heat Exchange stations, the intersection where heat is transferred from the transmission grid to the distribution grid. We treated each hear exchange station as a consumer and clustered according to their consumption pattern. The data in this study is kindly supplied by the district heating company AVA of Aarhus. 49 heat exchange stations were included in the analysis. The study has focused on clustering hourly consumption data for the entire month of January 2017. AVA expects that clustering of smart meter data, in time, can help improve production efficiency by $1-2 \%$, generating savings of 1.3-2.7 million euros a year and an unknown amount in capital expenditure. As mentioned in [2] clustering of electricity smart meter data has been performed at different time scales, from daily to a full year with hourly consumption recordings, the paper used hourly data for an entire month.

The most prevalent method for consumption clustering has been identified to be the K-Means algorithm, This paper has discussed the clustering performance and possible improvements to the algorithm. K-Means popularity among clustering algorithms can be attributed to its widespread implementation in popular analytical software, its stability and generally good performance. We used the python Sci-Kit Learning implementation of K-Means to generate the clustering, we scaled the input data to ensure pattern clustering rather than consumption volume clustering, and we applied four different cluster validation metrics for evaluating the optimum number of clusters. We found that K-Means clustering on scaled smart meter data to be academically viable, though the resulting clusters can be very unbalanced. This imbalance of the clusters is undesirable as it increases the risk of clustering outliers rather than clustering the general consumption structure. The identified clusters exhibit large dispersion resulting in overlapping clusters, a phenomenon also encountered in electricity meter clustering papers such as [3] rendering the practical applicability of the clustering less feasible. 
521 The clusters identified with autocorerlation features (ACF) are not merely subsets of the scaled clustering but 522 entirely different clusters identified in the data. Furthermore we performed a wavelet basis transformation of the original data. The ACF and Wavelet transformation enabled K-Means to account for autocorrelation while also dramatically reducing the dimension of the data. The ensuing clustering improved separation of clusters. The wavelet transformation resulted in nearly identical clusters as the scaling produces but did so at a five-fold reduction in dimension, significantly improving the K-Means clustering runtime..

A surprising result from the ACF clustering is that 5 out of 7 clusters have identical structure only offset by differences in coefficients values. This suggests that most of the heat exchange stations consumption pattern can be described by the same underlying time series model, regardless of composition of the consumers supplied by the individual HX stations. This finding is important for reducing capital expenditures when expanding the district heating grid. This is surprising as the demographic and consumer composition of an area intuitively is expected to influence the consumption pattern, however the clustering using ACF indicates this is not the case. As it is the first time it has been encountered further research is required in this area. Deeper knowledge of the different areas served by the HX stations could help in understanding the patterns observed.

Further improvements to this study include investigation into the stability of the clusters. Are the clusters stabile over time- or do the cluster members transition between clusters? For a study of these potential transitions and cluster stability over time, elimination of exoteric influences, such as; weather and temperature is needed.

Stability of the clustering was ensured by the concept of cross-validation. This paper has presented an adaptation of Cross-validation for unsupervised learning such as to give an indication of how much each cluster fluctuates. Cross-validation reduces the risk for overfitting the clusters to the data and functions as an evaluation of the validity of the cluster validation indices employed. Though there is not a simple unified approach for cross-validation in an unsupervised setting the paper [29] discusses cross-validation for principal components selection. In this paper we have applied leave-one-out cross-validation, utilizing validation indices as pseudo response variables for cross-validating the estimate of the optimum number of clusters.

\section{Conclusion}

This paper presents novel ways of transforming smart meter input data before applying K-Means clustering. The transformation can be regarded as feature extraction with subsequent clustering of the features rather than the original meter data. With success we empirically confirm the existence of autocorrelation in the meter data and are able to cluster the heat exchange stations with regard to their autocorrelation function. The effect of clustering the autocorrelation enables the K-Means algorithm to account for the autocorrelation inherent in the meter data. To the best of the authors' knowledge this transformation has not been performed on electricity meter data or district heat meter data before. The ability to cluster district heating consumption data enables utilities to optimize their production better, making for a more efficient use of fuels and a cheaper supply of heat in the current grid. Furthermore, the aggregated clusters identified in this paper can reduce capital expenses for grid expansions due to correct dimensioning. Finally the identification of homogeneous consumption clusters allow for consumption awareness campaigns for more conscious consumption. 
558 The resulting clustering when applying autocorrelation features generates more visually distinguishable 559 clusters. The wavelet transformation applied to the input data resulted in significant reduction in dimensions

560 while retaining the original structure of the data and produced similar clusters to the non-preprocessed 561 clustering. Both autocorrelation and wavelet transformations are efficient linear time algorithms that also 562 reduce dimensionality of the input dataset. This reduction in dimensionality significantly improves on the 563 worst-case running time of the overall clustering, while enabling K-Means to manage temporal dependencies. 564 We have selected balanced cluster solution as we are interested in clustering consumption groups rather than 565 individual consumption outliers.

566 However, we were unable to find significant differences in the clustering results. Unfortunately all cases 567 contain shadow clusters that are statistically indistinguishable from neighboring clusters. Apart from showing 568 that clever preprocessing of input data to K-Means can result in good cluster performance for the ACF 569 transformation, the reduction in upper bound runtime is reduced from $4^{744}$ to $7^{24}$ which is a significant 570 reduction in computational effort needed for the clustering process.

571 The main focus of this paper has been to apply the most prevalent clustering method from electricity 572 consumption clustering to district heating data. The literature concerning district heat consumption clustering 573 is still limited. This paper has proposed solutions to the K-Means algorithms limited ability to account for 574 autocorrelation by transformation of input data. We conclude that the K-Means algorithm is indeed capable of 575 clustering district heating consumption data. While diligently preprocessing the data we can further increase K576 Means applicability on more complex data structures, in this case enable accounting for auto-correlation. 
601

602

603

604

605

606

607

608

609

\section{Executive Summary}

* This paper clusters 49 district heat exchange stations applying smart meter consumption data, applying hourly recordings from entire January 2017, summing to 744 observations per station.

* K-Means was selected as clustering method due to its prevalence in the electricity consumption clustering literature.

$>$ Baseline clustering was done on normalized data, and validated using MIA, CDI, DBI and Silhouette index.

$>$ Autocorrelation was shown in the data and preprocessing of input enabled K-Means to account for autocorrelation information in the clustering.

- Autocorrelation feature extraction and Wavelets were applied to account for autocorrelation.

* K-Means was able to cluster district heat smart meter data.

$>$ The clustering was improved by preprocessing the data prior to clustering.

- Autocorrelation features enabled more detailed clusters

- Wavelet features enabled compression of input data with identical clusters as baseline.

* Preprocessing of input data can enable K-Means to account for different inherent data structures, improving overall clustering and worse-case runtime. The autocorrelation feature extraction developed finer clusters drastically improved the run-time.

\section{Acknowledgement}

The authors wish to thank AffaldVarme Aarhus for participating in the research project, by making relevant data available. Pia Thomsen and Rikke Brink $\varnothing$ Berg for valuable input and endless proof reading. This work is part of the CITIES (Centre for IT-Intelligent Energy Systems in Cities) research project.

This work was supported by the Danish Innovation Found. Grant DSF 1305-00027B (Det Strategiske Forskningsråd). The Danish Innovation Found had no involvement in the study design.

Declaration of interest: None. 
611 [1] Fjernvarme D. Fakta om fjernvarme 2017:1. http://www.danskfjernvarme.dk/presse/fakta-omfjernvarme (accessed March 16, 2018).

[2] Tureczek. Alexander M, Nielsen. Per S. Structured Literature Review of Electricity Consumption Classification Using Smart Meter Data. Energies 2017;10:584. doi:10.3390/en10050584.

615

616

617

[3] Viegas JL, Vieira SM, Melício R, Mendes VMF, Sousa JMC. Classification of new electricity customers based on surveys and smart metering data Commission for Energy Regulation. Energy 2016;107:804-17. doi:10.1016/j.energy.2016.04.065.

618

[4] Chicco G, Napoli R, Piglione F, Postolache P, Scutariu M, Toader C. Load pattern-based classification of 619 electricity customers. IEEE Trans Power Syst 2004;19:1232-9. doi:10.1109/TPWRS.2004.826810.

[6] Chicco G, Napoli R, Piglione F. Comparisons Among Clustering Techniques for Electricity Customer Basu K, Debusschere V, Douzal-chouakria A, Bacha S. Time series distance-based methods for nonintrusive load monitoring in residential buildings. Energy Build 2015;96:109-17. doi:10.1016/j.enbuild.2015.03.021.

Classification. IEEE Trans Power Syst 2006;21:1-7.

[7] Mcloughlin F, Duffy A, Conlon M. A clustering approach to domestic electricity load profile characterisation using smart metering data. Appl Energy 2015;141:190-9. doi:10.1016/j.apenergy.2014.12.039.

[8] Räsänen T, Voukantsis D, Niska H, Karatzas K, Kolehmainen M. Data-based method for creating electricity use load profiles using large amount of customer-specific hourly measured electricity use data. Appl Energy 2010;87:3538-45. doi:10.1016/j.apenergy.2010.05.015.

[9] Granell R, Axon CJ, Wallom DCH. Impacts of Raw Data Temporal Resolution Using Selected Clustering Methods on Residential Electricity Load Profiles. IEEE Trans Power Syst 2015;30:3217-24.

[10] Chicco G, Sumaili Akilimali J. Renyi entropy-based classification of daily electrical load patterns. IET Gener Transm Distrib 2010;4:736-45. doi:10.1049/iet-gtd.2009.0161.

[11] Park S, Ryu S, Choi Y, Kim J, Kim H. Data-Driven Baseline Estimation of Residential Buildings for Demand Response. Energies 2015;8:10239-59. doi:10.3390/en80910239.

[12] Tsekouras GJ, Hatziargyriou ND, Member S, Dialynas EN, Member S. Two-Stage Pattern Recognition of Load Curves for Classification of Electricity Customers 2007;22:1120-8.

[13] Carpaneto E, Chicco G, Napoli R, Scutariu M. Electricity customer classification using frequency - domain load pattern data 2006;28:13-20. doi:10.1016/j.ijepes.2005.08.017.

[14] Kang J, Lee J. Electricity Customer Clustering Following Experts' Principle for Demand Response Applications. Energies 2015;8:12242-65. doi:10.3390/en81012242.

[15] Haben S, Singleton C, Grindrod P. Analysis and Clustering of Residential Customers Energy Behavioral Demand Using Smart Meter Data. IEEE Trans Smart Grid 2015;7:136-44. 
660

661

662

663

664

665

666

667

668

669

670

671

672

673

674

675

676

677

doi:10.1109/TSG.2015.2409786.

[16] Gouveia JP, Seixas J. Unraveling electricity consumption profiles in households through clusters : Combining smart meters and door-to-door surveys. Energy Build 2016;116:666-76. doi:10.1016/j.enbuild.2016.01.043.

[17] Kavousian A, Rajagopal R, Fischer M. Determinants of residential electricity consumption : Using smart meter data to examine the effect of climate , building characteristics, appliance stock , and occupants ' behavior. Energy 2013;55:184-94. doi:10.1016/j.energy.2013.03.086.

[18] Mcloughlin F, Duffy A, Conlon M. Characterising domestic electricity consumption patterns by dwelling and occupant socio-economic variables : An Irish case study. Energy Build 2012;48:240-8. doi:10.1016/j.enbuild.2012.01.037.

[19] Ndiaye D, Gabriel K. Principal component analysis of the electricity consumption in residential dwellings 2011;43:446-53. doi:10.1016/j.enbuild.2010.10.008.

[20] Ozawa A, Furusato R, Yoshida Y. Determining the relationship between a household 's lifestyle and its electricity consumption in Japan by analyzing measured electric load profiles. Energy Build 2016;119:200-10. doi:10.1016/j.enbuild.2016.03.047.

[21] Gadd H, Werner S. Heat load patterns in district heating substations. Appl Energy 2013;108:176-83. doi:10.1016/j.apenergy.2013.02.062.

[22] Gianniou P, Liu X, Heller A, Nielsen PS, Rode C. Clustering-based analysis for residential district heating data. Energy Convers Manag 2018;165:840-50. doi:10.1016/j.enconman.2018.03.015.

[23] Cormen T, Rivest R, Leiserson C. Introduction to Algorithms. second edi. The MIT Press; 2001.

[24] Pedregosa F, Varoquaux G, Gramfort A, Michel V, Thirion B, Grisel O, et al. Scikit-learn: Machine Learning in Python. J Mach Learn Res 2012;12:2825-30. doi:10.1007/s13398-014-0173-7.2.

[25] Lattin J, Carrol JD, Green PE. Analyzing Multivariate Data. vol. 46. 1. st. Duxbury; 2004. doi:10.1198/tech.2004.s798.

[26] Bishop CM. Pattern Recognition and Machine Learning. 1st ed. Springer; 2006. doi:10.1117/1.2819119.

[27] López JJ, Aguado JA, Martín F, Mu F, Rodríguez A, Ruiz JE. Hopfield - K -Means clustering algorithm : A proposal for the segmentation of electricity customers 2011;81:716-24.

doi:10.1016/j.epsr.2010.10.036.

[28] Maulik U, Bandyopadhyay S. Performance evaluation of some clustering algorithms and validity indices. IEEE Trans Pattern Anal Mach Intell 2002;24:1650-4. doi:10.1109/TPAMI.2002.1114856.

[29] Perry PO. Cross-Validation for Unsupervised Learning 2009.

[30] Wasserman L. All of Statistics. vol. C. Springer; 2003. doi:10.1007/978-0-387-21736-9.

[31] Friedman J, Hastie T. The Elements of Statistical Learning. 1st ed. Springer; 2008. 
678 [32] Madsen H. Time Series Analysis. 1st ed. Chapman \& Hall/CRC; 2007.

679 [33] Warren Liao T. Clustering of time series data - A survey. Pattern Recognit 2005;38:1857-74.

680 doi:10.1016/j.patcog.2005.01.025.

681

682

683

684

685

686

[34] Barford LA, Fazzio RS, Smith DR. An introduction to wavelets. Hewlett-Packard Labs, Bristol, UK, Tech Rep HPL-92-124 1992;2:1-29. doi:10.1109/99.388960.

[35] Morchen F. Time series feature extraction for data mining using DWT and DFT 2003:1-31. doi:citeulikearticle-id:3973352.

[36] Li T, Li Q, Zhu S, Ogihara M. A survey on wavelet applications in data mining. ACM SIGKDD Explor Newsl 2002;4:49-68. doi:10.1145/772862.772870.

687

[37] Nason GP. Wavelet Methods in Statistics with R. 2008. doi:10.1007/978-0-387-78171-6.

688

[38] Graps A. An introduction to wavelets. IEEE Comput Sci Eng 1995;2:50-61. doi:10.1109/99.388960.

689 [39] Wasilewski F. PyWavelets 2006.

690 [40] Johnson RA. Probability and Statistics for Engineers. 6th ed. Miller \& Freund's; 2000.

691 [41] Jones E, Oliphant T, Peterson P. SciPy: Open Source Scientific Tools for Python 2001.

692 [42] Arthur D, Vassilvitskii S. How slow is the k-means method? Proc Twenty-Second Annu Symp Comput

693

Geom - SCG '06 2006:144. doi:10.1145/1137856.1137880. 